\title{
Low-Temperature Characteristics of Well-Type Guard Rings in Epitaxial CMOS
}

\author{
Chih-Yao Huang, Member, IEEE, Ming-Jer Chen, Member, IEEE, Jeng-Kuo Jeng,
} and Ching-Yuan $\mathrm{Wu}$, Member, IEEE

\begin{abstract}
Characterization and simulation of minority-carrier well-type guard rings in epitaxial substrate at $77 \mathrm{~K}$ were performed and compared with those at RT. The escape probability in a narrow guard-ring structure under the same amount of minority carrier injection increases by about one order of magnitude when temperature decreases to $77 \mathrm{~K}$. This degradation in the guard-ring efficiency can be attributed to the enhanced drift mechanism in the conductivity-modulated layer between the well bottom junction and the epitaxial high/low junction at 77 $K$. In contrast, this mechanism enhances the width dependence of the escape probability at $77 \mathrm{~K}$. The higher minority-carrier recombination velocity of the epitaxial high-low junction contributes to the stronger width dependence secondarily. When the epitaxial layer thickness becomes thinner, the simulation also demonstrates a stronger width dependence of the escape current as well as a reduction in its magnitude. A lightly-doped epitaxial layer on a heavily-doped substrate exhibits even more importance in the guard ring efficiency for low temperature operation, and its thickness should be kept as thin as possible.
\end{abstract}

\section{INTRODUCTION}

$\mathbf{L}$ OW-TEMPERATURE CMOS has been extensively studied as a result of more excellent performance and reliability than RT operation at device, circuit, and system levels [1]-[4]. Its advantages include higher carrier mobility, higher switching speed, sharper subthreshold slope, decreased noise and junction leakage current, reduced parasitic resistance and interconnection resistance, and better latch up immunity.

On the other hand, minority-carrier well-type guard rings at RT have been studied recently [7]-[12]. Minority-carrier well-type guard rings, which are regarded as one of the most effective methods for latch up elimination, have been widely used for collecting minority carrier injection from parasitic emitter structures around I/O area in CMOS technology. The efficiency of such guard ring can be greatly improved by using epitaxial layer substrate [8]. The mechanism responsible for this enhancement has been reported to be Auger recombination in the heavily-doped substrate [9]. An analytic design formulation has been proposed to quantitatively describe guard ring efficiency and applied to the establishment of design guideline for minimum area consumption [10]-[12]. In view

Manuscript received November 20, 1995; revised April 11, 1996. The review of this paper was arranged by Editor K. Tada. This work was supported by the National Science Council under Contract NSC 84-2215-E-009-043.

C.-Y. Huang is with Mosel Vitelic Inc., Hsinchu 300, Taiwan, R.O.C.

M. J. Chen and C.-Y. Wu are with the Institute of Electronics, National Chiao-Tung University, Hsinchu 300, Taiwan, R.O.C.

J.-K. Jeng is with Vanguard International Corporate, Hsinchu 300, Taiwan, R.O.C.

Publisher Item Identifier S 0018-9383(96)08641-8. of the excellent features of low-temperature CMOS, the lowtemperature operation of the guard ring is clearly worth more investigation. In this work we present both measured characteristics and numerical simulations of guard rings with different guard-ring width at $77 \mathrm{~K}$ as compared with those at RT. The physical mechanisms contributing to the behavior of the guard ring are discussed in detail by means of twodimensional numerical analysis. Finally design considerations for improved low-temperature performance will be addressed based on the experiments and simulations.

\section{EXPERIMENTAL ReSUlTS}

The schematic top view and cross section along the line $A A^{\prime}$ of the test structure investigated is shown in Fig. 1. The inner/outer n-well collectors surround an emitter region on an epitaxial heavily-doped substrate fabricated by $1.0 \mu \mathrm{m}$ n-well polysilicon CMOS technology. The inner square collector is a guard ring and the outer collector acts as internal circuitry. The emitter region represents drain/source diffusion in a MOS device. The four different guard ring widths $W_{g}$ are 6,20 , $40,60 \mu \mathrm{m}$ and the epitaxial-layer thickness is about $10.0 \mu \mathrm{m}$. The emitter junction depth and n-well junction depth are 0.3 and $3.0 \mu \mathrm{m}$, respectively. The peak doping concentrations in the $\mathrm{n}^{+}$emitter, $\mathrm{n}$-well, $\mathrm{p}$-type epitaxial layer, and $\mathrm{p}^{+}$ substrate are $2 \times 10^{19}, 6 \times 10^{16}, 2 \times 10^{15}$, and $4 \times 10^{18}$ $\mathrm{cm}^{-3}$, respectively. The devices were mounted on 18-pin ceramic dual-in-line packages. The package was put into a RMC-Cryosystems LTS-22 closed-cycle cold head for lowtemperature measurement. Good thermal contact has been made for effective cooling. Besides being measured by a silicon diode temperature sensor, the device temperature was also extracted and checked by $\mathrm{PN}$-junction diffusion equation $I_{o} \exp (q V / n K T)$. The $I-V$ characteristics were measured with both wells biased at $5.0 \mathrm{~V}$, the emitter grounded, and the base-emitter bias $V_{b e}$ swept from 0.0 to $2.0 \mathrm{~V}$ for both the RT and $77 \mathrm{~K}$ cases.

Fig. 2(a) shows the Gummel plot of the emitter current $I_{e}$, collection current $I_{c 1}$, base current $I_{b}$, and escape current $I_{c 2}$ for a $6 \mu \mathrm{m}$ wide guard ring at both RT and $77 \mathrm{~K}$. The turn-on voltage in Fig. 2(a) obviously increases by about 0.4 $\mathrm{V}$ at $77 \mathrm{~K}$ compared with the RT case. This is due to the weak increase of the PN junction built-in potential at low temperature [15]. Low-temperature operation of a guard ring seems to improve its performance in ideal diffusion region. However, $0.4 \mathrm{~V}$ increase will not always be sufficient for latch up elimination. This is only a rather limited improvement 


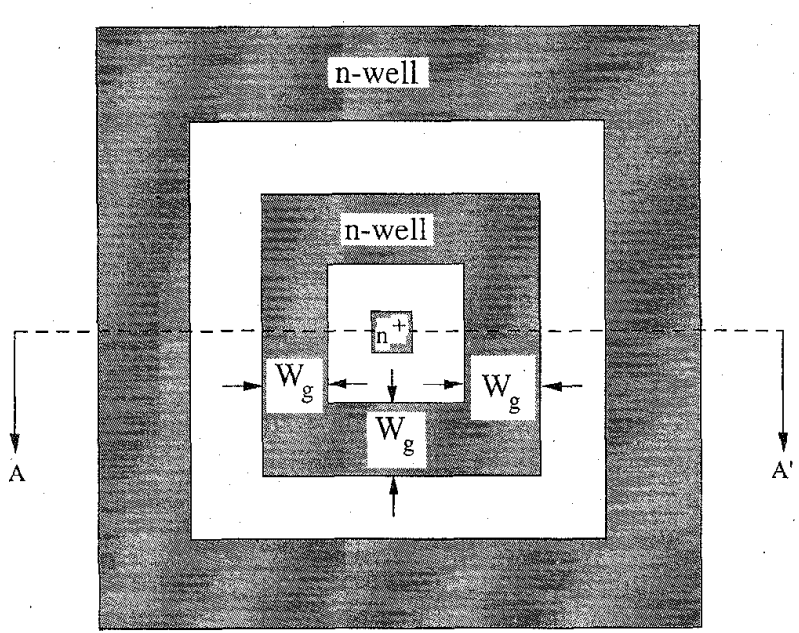

(a)

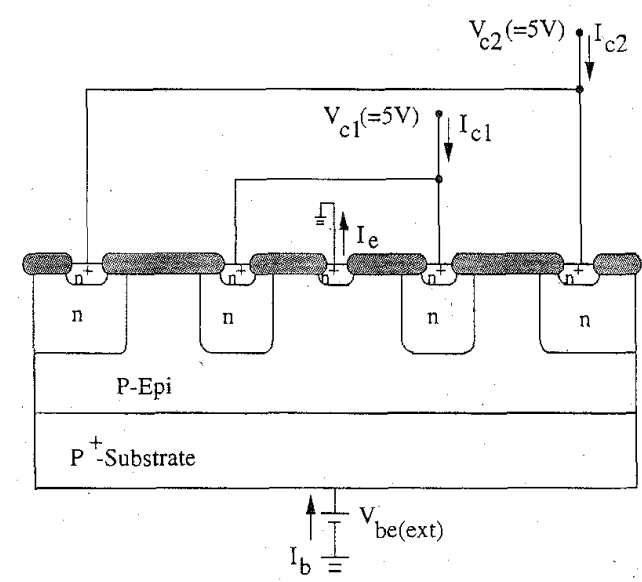

(b)

Fig. 1. Schematic top view and cross section along the line $A A^{\prime}$ of n-well guard ring structure on $\mathrm{p}$-epi/ $\mathrm{p}^{+}$-substrate where $\mathrm{n}^{+}$emitter, inner, and outer n-well collectors are also shown.

as a consequence of high-level injection during latch up state. In various circumstances the place most sensitive to latch up in an IC is the $\mathrm{I} / \mathrm{O}$ region. Large current flow and large parasitics may be present in such regions. Abnormal circuit voltages, which are immense especially in electrostatic discharge (ESD) event, may be encountered [6]. Devices suffering from outer noise in these regions may be triggered into on state and inject minority carriers into either the substrate or the well, potentially triggering other pnpn paths nearby. Thus the quantity of minority carriers injected instead of the applied outer voltage represents a key factor in effective evaluation of latch up susceptibility around the I/O areas [7]. Hence the intuitively applied bias point of view should be improved, and more insights can be obtained by means of the comparison under the same amount of the injected minoritycarrier density from the parasitic emitter, namely the equal $I_{e}$. In Fig. 2(a), some typical $V_{b e}$ biases at RT for comparison may arbitrarily be selected: for instance, $0.7,1.0$, and $1.2 \mathrm{~V}$. The corresponding emitter currents are directly obtained from the RT Gummel plot. Then three bias values are obtained with the same emitter current values in the $77 \mathrm{~K}$ Gummel plot: 1.1, 1.4 , and $1.8 \mathrm{~V}$, respectively. That is, the emitter currents of $1.1,1.4$, and $1.8 \mathrm{~V}$ at $77 \mathrm{~K}$ are identical to those of $0.7,1.0$, and $1.2 \mathrm{~V}$ at RT, respectively. Both $0.7 \mathrm{~V}$ at RT and $1.1 \mathrm{~V}$ at $77 \mathrm{~K}$ represent the onset of the high-level injection of the Gummel plot in this structure.

Based on the above method, the escape probability was calculated from Fig. 2(a) by the ratio of the escape current divided by the emitter current, $I_{c 2} / I_{e}$, which is the transport factor from the emitter to the outer n-well when the guard ring is also collecting carriers [8]. This quantity expresses the amount of injected minority carriers that escape from the guard ring collection and into the substrate. In addition, $I_{c 1} / I_{e}$, which is the transport factor from the emitter to the guard ring n-well, was also calculated and compared. Either $\left(1-I_{c 2} / I_{e}\right)$ or $I_{c 1} / I_{e}$ can be understandable as a measure of guard-ring efficiency. However, the best indication is the escape probability itself since a very small change in the $\left(1-I_{c 2} / I_{e}\right)$ or $I_{c 1} / I_{e}$ may produce a change of several orders of magnitude in the $I_{c 2} / I_{e}$. As the $I_{c 2} / I_{e}$ becomes larger the guard-ring efficiency reduces more. On the contrary, as the $I_{c 2} / I_{e}$ becomes smaller the guard-ring efficiency improves more. The escape probability for a $6 \mu \mathrm{m}$ wide guard ring at $77 \mathrm{~K}$ was found to be about ten times higher than that at RT, as illustrated in Fig. 2(b), yet $I_{c 1} / I_{e}$ remains almost the same for both cases. This result demonstrates the reduced efficiency of the narrow guard ring at $77 \mathrm{~K}$. Fig. 2(c) shows the plot of the escape probability as a function of $W_{g}$ at both RT and 77 K. In Fig. 2(c) the applied $V_{b e}$ biases for the characteristics at RT and $77 \mathrm{~K}$ are separately $0.9 \mathrm{~V}$ and $1.3 \mathrm{~V}$ for the first pair of comparison, and $1.2 \mathrm{~V}$ and $1.9 \mathrm{~V}$ for the second pair. The measured results at these different biases corresponding to the same amount of the injected emitter currents at the two specified temperatures. The width dependence of both the $I_{c 2}$ and $I_{c 2} / I_{e}$ becomes stronger as the temperature is lowered. In addition, the amount of the $I_{c 2}$ at $77 \mathrm{~K}$ with $W_{g}$ around $6 \mu \mathrm{m}$ becomes at least one order of magnitude larger than that at RT. The curves at $77 \mathrm{~K}$ show a rise in the $I_{c 2}$ and $I_{c 2} / I_{e}$ with narrow $W_{g}$ and a reduction with large $W_{g}$ as compared with those at RT. The cross points of the RT and $77 \mathrm{~K}$ lines are located at $W_{g}$ ranging from 20 to $35 \mu \mathrm{m}$, depending on the amount of the applied biases. To sum up, these results indicate that the narrow guard ring has reduced collection efficiency whereas the wide guard ring has improved efficiency for low temperature operation. Moreover, the width dependence becomes gradual as the $V_{b e}$ increases.

\section{REDUCED COLLECTION EFFICIENCY IN NARROW GUARD RINGS}

Because of the wide spreading of minority carriers over the whole substrate and the complicated nature in many physical models at low temperature, it is necessary to employ 2-D numerical simulation for detailed analysis. Twodimensional numerical simulation utilizing a device simulator MEDICI [16] was performed for both the RT and $77 \mathrm{~K}$ cases. Because the test structure and its current flow pattern are symmetrical, only the right half of the device in Fig. 1 was 


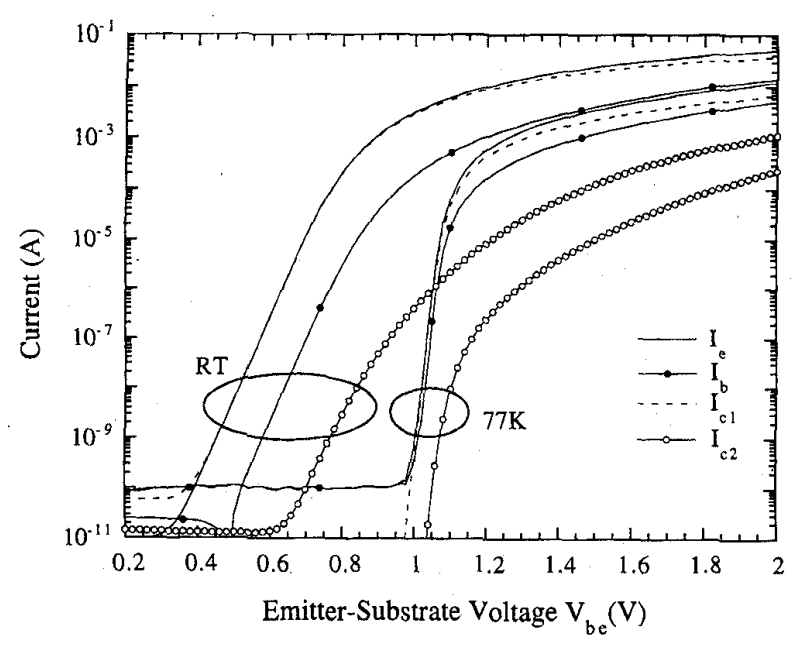

(a)

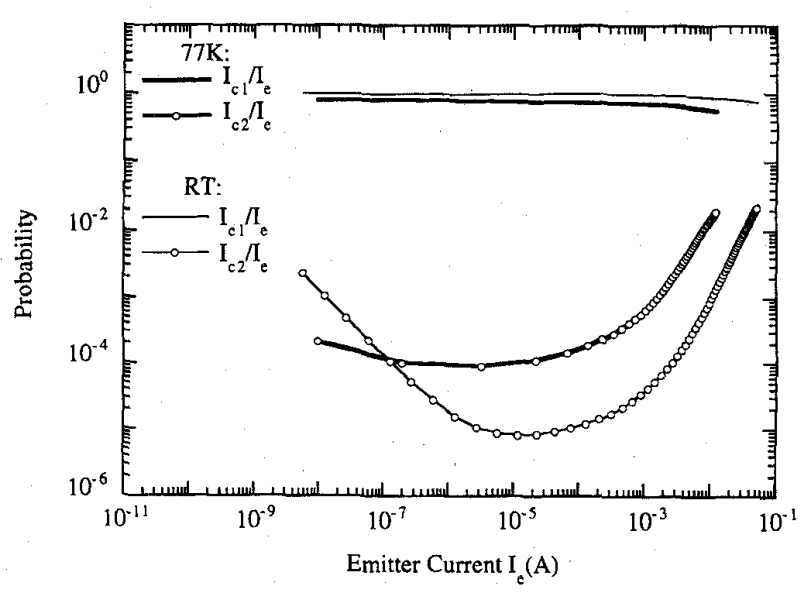

(b)

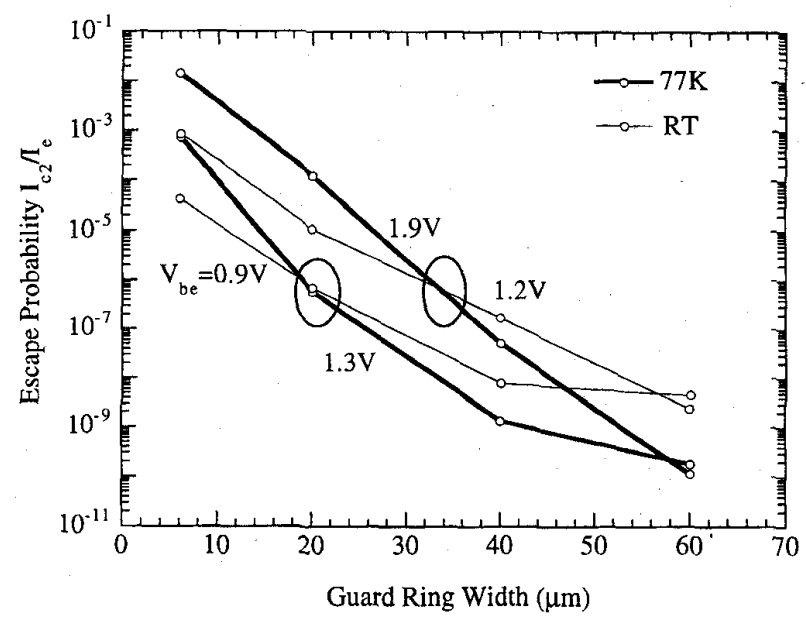

(c)

Fig. 2. (a) Measured characteristics of the emitter current $I_{e}$, collection current $I_{c 1}$, base current $I_{b}$, and escape current $I_{c 2}$ for a $6 \mu \mathrm{m}$ wide guard ring at RT and $77 \mathrm{~K}$ with both wells biased at $5 \mathrm{~V}$, emitter grounded, and emitter-substrate bias swept from 0.0 to $2,0 \mathrm{~V}$. (b) Comparison of calculated ratio of collection current divided by emitter current $I_{c 1} / I_{e}$ and escape probability (defined by the ratio of the escape current divided by the emitter current $I_{c 2} / I_{e}$ ) versus emitter current $I_{e}$ at RT and $77 \mathrm{~K}$. (c) The measured escape probabilities $I_{c 2} / I_{e}$ as a function of $W_{g}$ at RT and $77 \mathrm{~K}$. The $V_{b e}$ biases of the first comparison pair are 0.9 and $1.3 \mathrm{~V}$, and the second pair are 1.2 and $1.9 \mathrm{~V}$ at RT and $77 \mathrm{~K}$, respectively.

simulated. The simulator in this work, however, is only used for qualitative analysis of low-temperature applications. This is due to simplicity of the low-temperature models and their coefficients, and the nonuniform current distribution along the perimeter of the square layout in the test pattern. For the low-temperature simulation the default models built in the simulator could not completely satisfy the requirements [17], [18]. Among the models utilized in this work, carrier freezeout with Fermi-Dirac statistics is the basic phenomenon at low temperature [14]. Because of the difficulty in introducing the temperature-dependent freezeout ionization energies in the incomplete ionization [18] into the simulator, the two energy values were estimated from the experimental data in [14]. For temperature-dependent energy gap, the default model in the simulator was still adopted in this work. Although the built-in model was used, it was reported that the effect of different model is not significant in qualitative analysis [18]. For SRH recombination, a fully justified model is still unavailable [17], [18]. Hence, the model equation and parameter values at RT were still used here for the $77 \mathrm{~K}$ study [19]. Other models include Fermi-Dirac statistics, density of states for conduction and valence band [17], temperature-dependent bandgap narrowing effect [21], Auger recombination [22], carrier mobility with concentration, temperature and field dependence [23] and [24] as well as temperature-dependent carrier saturation velocity [14]. The coefficients in some of these models were modified to meet the requirements at low temperature. Moreover, the nonuniform current distribution along the square test-pattern perimeter is actually beyond the capability of a 2-D simulator. The high applied bias and low temperature further lead to a more complex situation in the current distribution. Under such circumstances, the simulator is only feasible for investigating qualitative behaviors and giving more physical insights for experiment phenomena. 


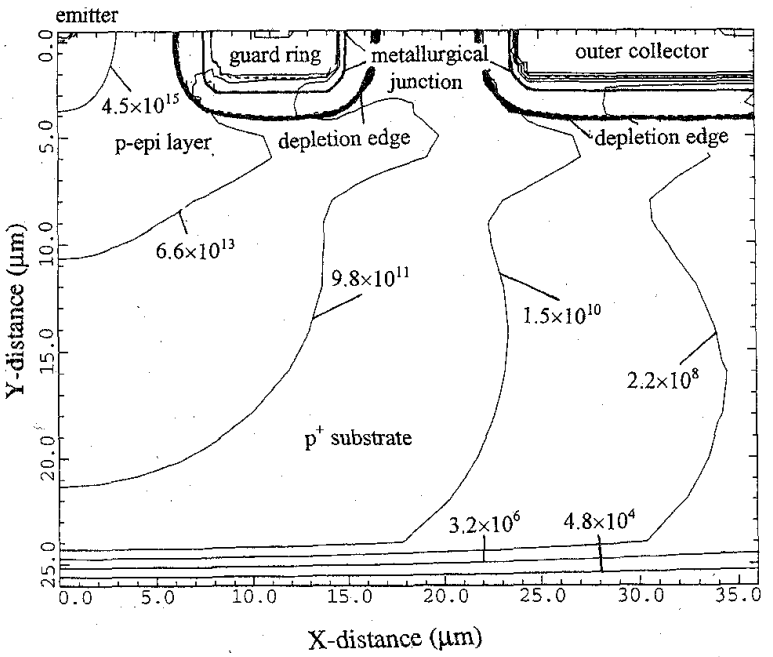

(a)

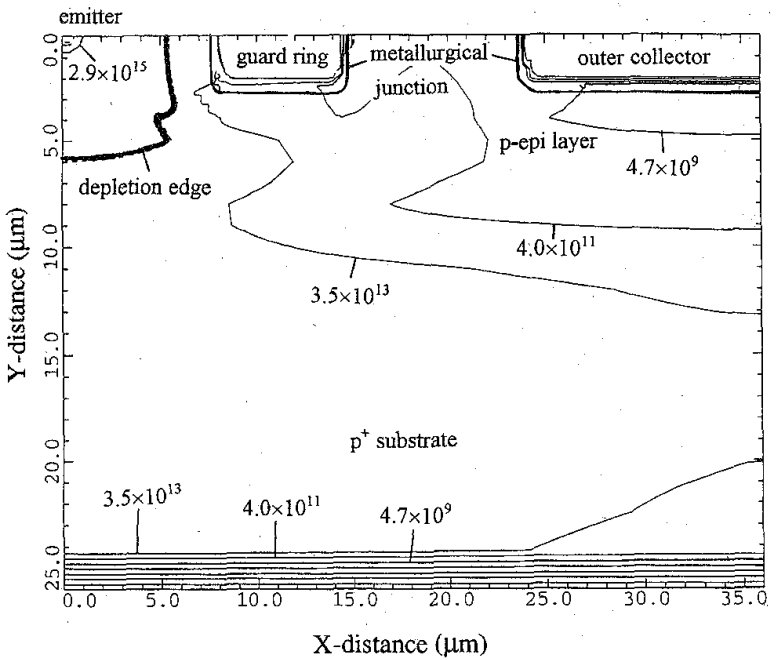

(b)

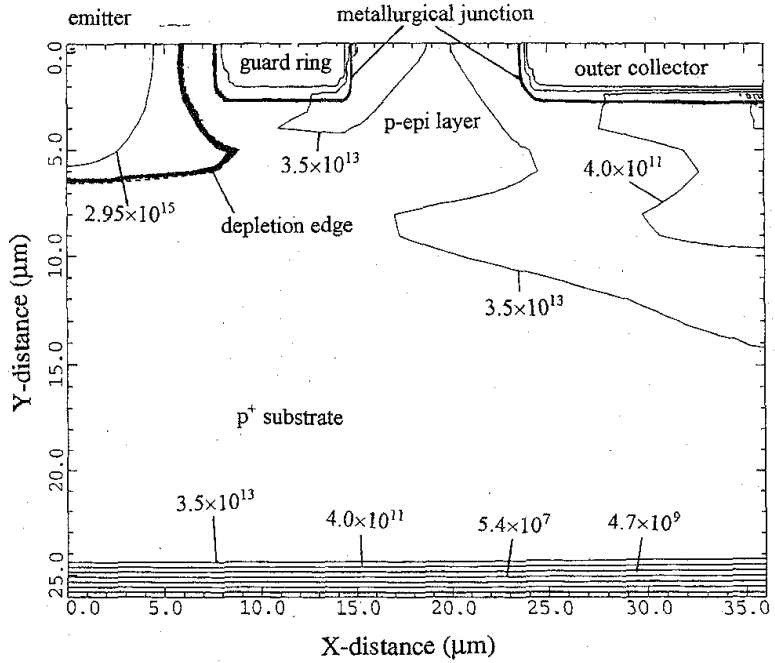

(c)

Fig. 3. 2-D minority carrier concentration contour: (a) at $V_{b e}=0.7 \mathrm{~V}$ for RT, (b) at $V_{b e}=1.1 \mathrm{~V}$ for $77 \mathrm{~K}$, and (c) at $V_{b e}=1.4 \mathrm{~V}$ for $77 \mathrm{~K}$. The two bold lines in (a) around the two wells represent the depletion region edges. The bold lines in (b) and (c) have merged into a single line and shifted to the intrinsic region around the parasitic emitter.

Fig. 3(a) shows the 2-D minority-carrier concentration contour for $V_{b e}=0.7 \mathrm{~V}$ at RT, while Fig. 3(b) and (c) for $V_{b e}=$ $1.1 \mathrm{~V}$ and $1.4 \mathrm{~V}$ at $77 \mathrm{~K}$, respectively. The depletion region edges at RT around both wells are smoothly parallel to the metallurgical junctions with the same shape, whereas at 77 $\mathrm{K}$ they are merged together in the area between the two $n-$ wells and spread over the whole substrate, as indicated by the bold line in Fig. 3(b) and (c). Hence, the electric field is apparently distributed more widely at $77 \mathrm{~K}$ than RT. That is, low-temperature operation can be expected to cause more severe conductivity modulation after high-level injection, or equivalently high injection condition will be easily obtained by a smaller amount of minority carrier injection as a result of majority carrier freezeout, as reported in [5], [18], and [20]. At low temperatures the depletion region of a $\mathrm{PN}$ junction generally extends slightly, in this specific circumstance the number of mobile holes are not easy to increase near the emitter region due to freezeout, and hence holes which come from the very far $n$-well depletion edge increase the depletion region rapidly to form an almost fully depleted lightly-doped layer. More minority carrier contour lines gather near the bottom of the substrate and spread more deeply into the substrate, implying that more minority carriers have penetrated into the heavily-doped substrate and that the current in the heavily-doped substrate has become larger accordingly.

According to the recent studies [10], [11], the escape current $I_{c 2}$ is composed of two major components: the first component is due to the minority carriers injected into the sandwich layer between the well bottom junction and the epitaxial high/low junction, namely the sandwich current $I_{\text {san }}$; and the second is due to the minority carriers penetrating the high/low junction and spreading out in the large, heavily-doped substrate, namely the substrate current $I_{s u b}$. Ordinarily the first component dominates the second especially for narrow guard 
ring cases. At RT the $I_{\mathrm{c} 2}$ equals $I_{\text {san }}$ for narrow $W_{g}$ while it becomes nearly the $I_{s u b}$ for wide $W_{g}$ [12]. In contrast, at low temperature the $I_{c 2}$ totally equals the $I_{s a n}$ for thicker epitaxial layer whereas it becomes the $I_{s u b}$ component flowing in the high/low junction transition region as in Fig. 5(b) for thinner epitaxial layer [12]. The $I_{s u b}$ deep into the substrate corresponds to the $I_{b}$ in most cases at low temperature. These results will be discussed in the following sections. At highlevel injection regime of the parasitic bipolar devices, the concentration of the minority carriers becomes comparable to that of the majority carriers, and both the diffusion and drift mechanisms should be considered for the $I_{s a n}$. In the respect of the diffusion mechanism, its temperature dependence can be expressed as [13]

$$
J_{d i f f}=K T^{3} \exp \left(-\frac{E_{g}}{K T}\right)\left[\exp \left(\frac{q V}{K T}\right)-1\right] .
$$

This temperature dependence is more than merely exponential change, in fact, diffusion current decreases by half for every $6^{\circ} \mathrm{K}$ decrease in temperature. Therefore, diffusion current at $77 \mathrm{~K}$ should decrease by a rather substantial amount. On the other hand, the drift component may increase due to enhanced mobility in moderately-doped semiconductors and/or expansion of electric field from 300 to $77 \mathrm{~K}$. As for carrier mobility, it is well known that the temperature dependence of lattice scattering is generally proportional to $\mathrm{T}^{-3 / 2}$. When temperature is lowered to $77 \mathrm{~K}$, lattice scattering weakens and therefore carrier mobility increases. In our test structure the doping of the sandwich layer where most of the escape current flows is $2 \times 10^{15} \mathrm{~cm}^{-3}$. Mobility enhancement plays an important role undoubtedly. In addition to the mobility factor, the depletion region of the well bottom junction covers most of the sandwich layer as revealed by the numerical simulation. The electric field extends over nearly all of this district where most of the escape current flows. This field factor also contributes to a larger drift component. As regards the second escape current component in the heavily-doped substrate, the electric field also penetrates into this region. Hence the drift current at $77 \mathrm{~K}$ can still be expected to be larger than that at RT. Almost all the substrate current flows downward to the substrate contact as shown in Fig. 3(b) and (c) as well as the 2-D current-flow vector plot. As a result, the increased escape current should result from the enhanced drift component along the conductivity-modulated sandwich layer.

The drift current of minority carriers can be calculated by $q n \mu E$ with the electric field and the minority carrier concentration data along a specific line in the test structure from the simulated solutions. Minority carrier mobility $\mu$ is calculated by utilizing the same model used in the simulations. The calculated results for comparison between $0.7 \mathrm{~V}$ at RT and $1.1 \mathrm{~V}$ at $77 \mathrm{~K}$ are shown in Fig. 4(a)-(d). Fig. 4(a) and (b) illustrate the electric field distribution and electron drift current density, respectively, in the $X$-direction along a horizontal line at $Y=4.5 \mu \mathrm{m}$ within the sandwich layer. The $X$-direction electric field at $77 \mathrm{~K}$ is very similar to that at RT except in the range from $13.5-25 \mu \mathrm{m}$, which is located between the two n-wells. In this district, the electric field at $77 \mathrm{~K}$ is much larger than that at RT. This confirms the wide distribution of the electric field over the sandwich layer at 77 K. In Fig. 4(b) the $X$-direction minority-carrier drift current density at low-temperature is larger than at RT along the entire line. The drift current density between the two n-wells, most of which flows directly into the outer n-well, is about 500 times greater at $77 \mathrm{~K}$ than that at RT. It is still about one order of magnitude greater under the outer n-well. Note that although the electric field under the outer n-well is nearly identical for both temperatures, the low-temperature current density is still greater than that at RT. The 2-D simulation results also indicate almost the same minority-carrier concentration for both cases, which verifies that mobility is enhanced at low temperature. Fig. 4(c) and (d) again, represent the electric field and electron drift current density, respectively, in the $Y$-direction along the same line. Once more the electric field is quite similar along the whole range except that between the two wells. In this case the electric field at $77 \mathrm{~K}$ is indeed smaller than that at RT. Nevertheless, at a distance farther than $25 \mu \mathrm{m}$, which is the region beneath the outer n-well, the drift current density in the $Y$-direction at $77 \mathrm{~K}$ is still greater. This fact again verifies the argumert that mobility is enhanced.

The comparison addressed is further changed to a vertical line at $X=20 \mu \mathrm{m}$ along the outer $\mathrm{n}$-well sidewall. The results are shown in Fig. 4(e)-(h). The electric field and electron drift current in the $X$-direction are plotted in Fig. 4(e) and (f), respectively. The electric field at $77 \mathrm{~K}$ exhibits a more uniform distribution and is higher than that at RT along the whole depth of the substrate. Similar to the electric field, the electron drift current in the $X$-direction at $77 \mathrm{~K}$ is more uniform and over 500 times higher than that at RT. Here a larger portion of the current spreads into the heavily-doped substrate, where it becomes comparable in quantity to that in the sandwich layer. The current level at $77 \mathrm{~K}$ with depth less than $6.0 \mu \mathrm{m}$ is clearly larger. Fig. $4(\mathrm{~g})$ and (h) compare the electric field and electron drift current in the $Y$-direction along the same line. Once more the electric field at $77 \mathrm{~K}$ is distributed more uniformly into the heavily doped substrate and is weaker than that at RT in the depth range between 5.0 and $12.0 \mu \mathrm{m}$. This is due to the decrease of the built-in field across the $\mathrm{p} / \mathrm{p}^{+}$transition junction, which will be discussed later. Nevertheless, the $Y$-direction electron drift current at RT is smaller than that at $77 \mathrm{~K}$ along the entire line. Not only the quantity of the current density below the high/low junction but also its uniform distribution throughout the substrate at $77 \mathrm{~K}$ are clearly observed. Therefore, the escape current $I_{c 2}$ at $77 \mathrm{~K}$ is larger than at RT due to drift component enhancement in the conductivity-modulated layer. The above discussions compare $0.7 \mathrm{~V}$ at RT and $1.1 \mathrm{~V}$ at $77 \mathrm{~K}$, both of which represent the onset of high-level injection at two temperature. Conductivity modulation is obviously more significant and escape current can also be expected to be larger at low temperature for higher $V_{b e}$ bias. This can be observed in Fig. 3(c) that the minority carrier contours spread more deeply into the large substrate.

\section{WIDTH-DEPENDENT CHARACTERISTICS}

The simulation analysis now concentrates on the widthdependent behaviors of the guard ring. The simulated minority- 


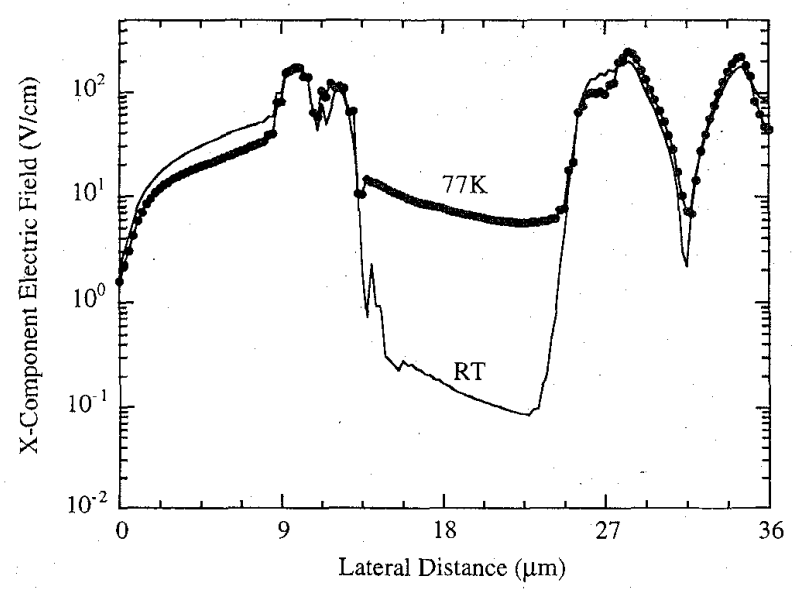

(a)

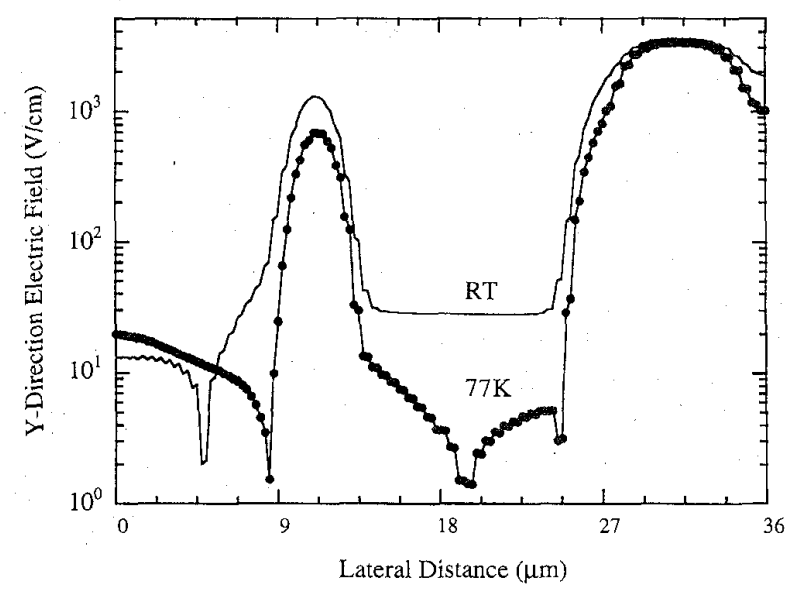

(c)

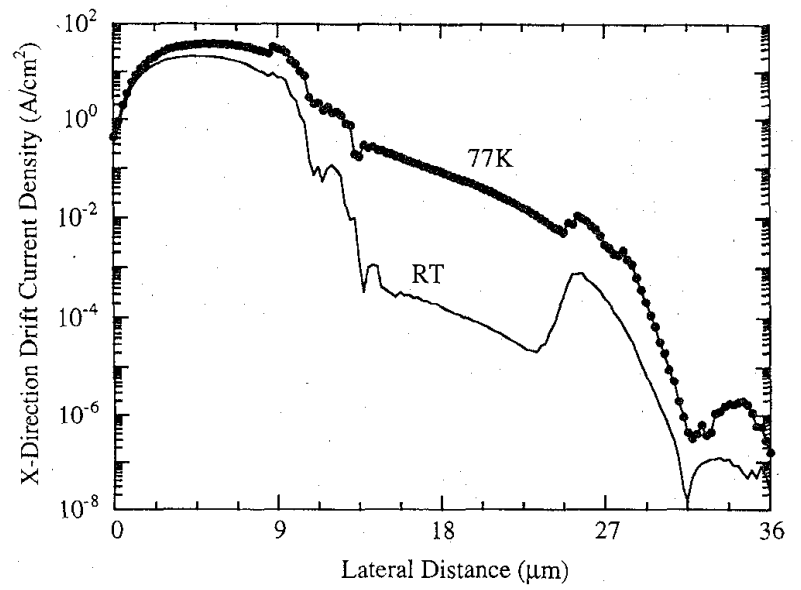

(b)

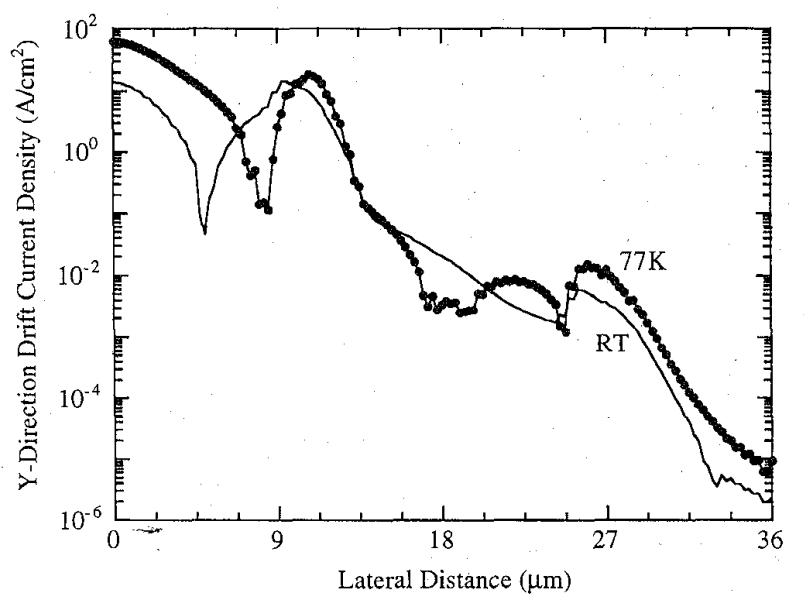

(d)

Fig. 4. Comparison of electric field distribution: (a) in the $X$-direction and (c) in the $Y$-direction between $V_{b e}=0.7 \mathrm{~V}$ at $\mathrm{RT}$ and $V_{b e}=1.1 \mathrm{~V}$ at 77 $\mathrm{K}$ along a horizontal line at $Y=4.5 \mu \mathrm{m}$ deep in the sandwich layer of the test structure. Comparison of calculated electron drift current density between $V_{b e}=0.7 \mathrm{~V}$ at RT and $V_{b e}=1.1 \mathrm{~V}$ at $77 \mathrm{~K}$ along this line (b) in the $X$-direction and (d) in the $Y$-direction.

carrier concentration contours and their current-flow vectors with $W_{g}=40 \mu \mathrm{m}$ at RT and $77 \mathrm{~K}$ are shown in Fig. 5(a)-(d). The depletion region spreads out over the whole epitaxial sandwich layer the same as the narrow guard ring case, while the depletion regions at RT retain the shapes of the n-wells. The depletion region extends the influence of the reversebiased electric field deeply into the sandwich layer. The electric field can sweep the minority carriers of the $I_{\text {san }}$ into the guard ring bottom, thus improving the collection capability of the guard ring, i.e., stronger width dependence of the $I_{c 2}$. It is important to note that the depletion region all over the sandwich layer also contributes to the enhancement of the drift component flowing toward the outer n-well. Therefore, the two competitive tendencies combine into the present result, for a narrow guard ring its bottom junction is not long enough to collect most of the minority carriers in the sandwich layer. The $I_{c 2}$ at $77 \mathrm{~K}$ is dominated by the enhanced drift component and thus larger than that at RT. In contrast, for wide guard ring its bottom junction is long enough to capture most part of the minority carriers and few carriers can reach the distant outer well, i.e., the $I_{c 2}$ at $77 \mathrm{~K}$ is smaller than that at RT. As illustrated in Fig. 5(b), the $I_{c 2}$ is actually composed of only the minority carriers flowing along the high/low junction transition region and the $I_{s a n}$ is completely absorbed by the n-well guard ring. The current vectors in the heavily-doped substrate also point toward the substrate contact completely. On the other hand, the current vectors of $I_{c 2}$ at RT all come from the heavily-doped substrate as shown in Fig. 5(d) and only a portion of the current vectors in the substrate flow toward the bottom contact. Fig. 5(a) and (c) also reveal such deeper penetration of the minority-carrier contours at $77 \mathrm{~K}$ than that at RT. Thus the $I_{c 2}$ at $77 \mathrm{~K}$ exhibits strong width dependence because they flow along the high/low transition region which is still strongly controlled by the guard ring as in [12]. In contrast, the $I_{c 2}$ at RT all come from the substrate, so the controllability of the guard ring becomes weak.

Normally, an epitaxial layer on a highly-doped substrate is the most effective structure for enhanced guard ring per- 


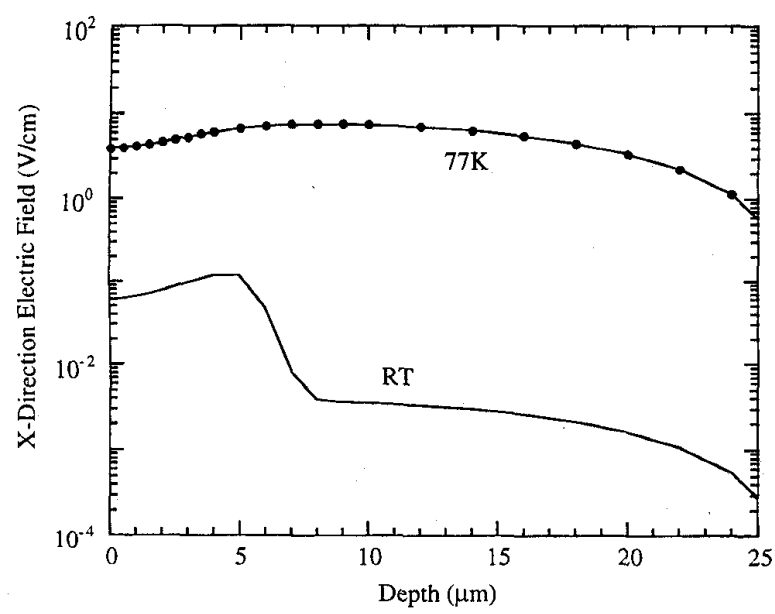

(e)

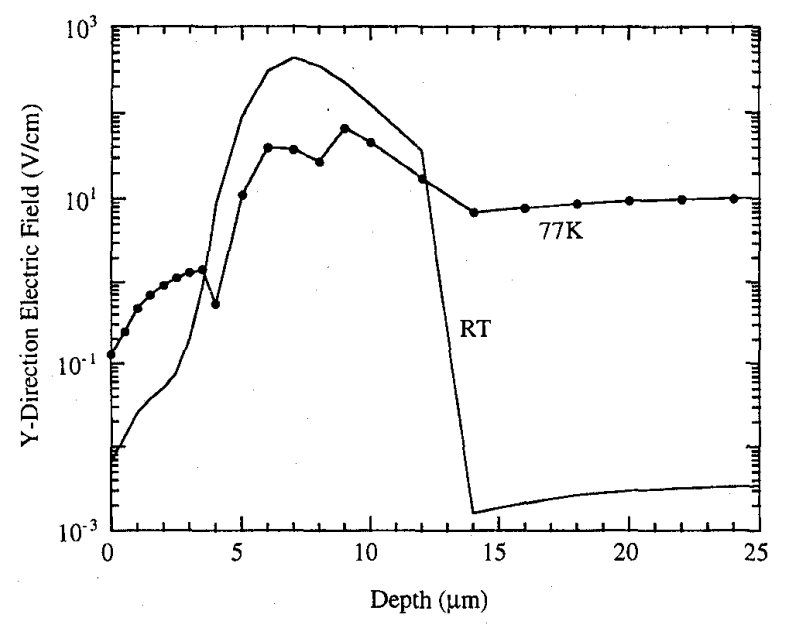

(g)

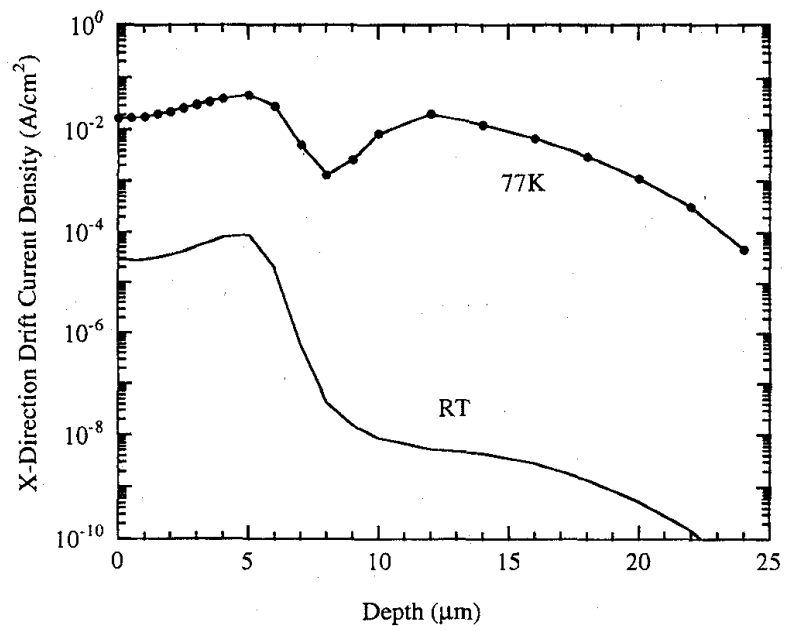

(f)

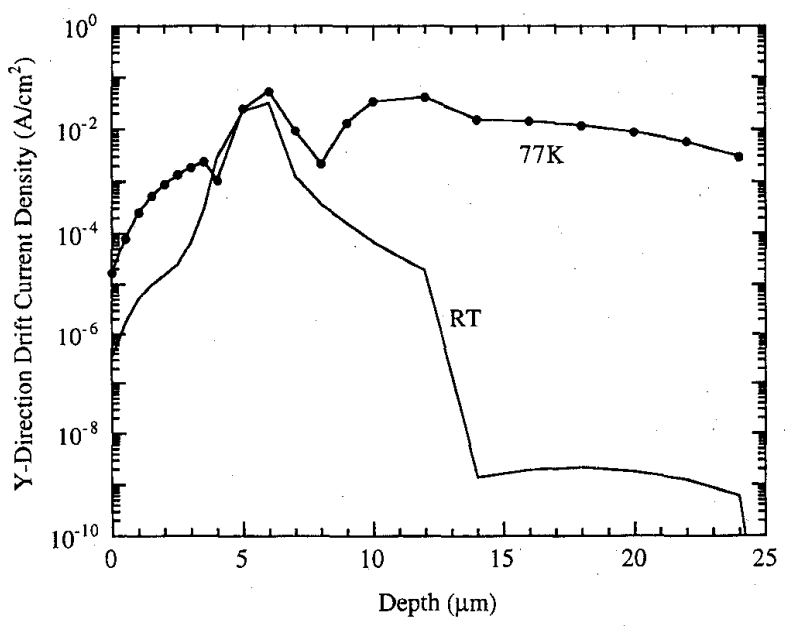

(h)

Fig. 4. (Continued). Comparison of electric field distribution. (e) in the $X$-direction and $(\mathrm{g})$ in the $Y$-direction between $V_{b e}=0.7 \mathrm{~V}$ at $\mathrm{RT}$ and $V_{b e}=1.1$ $\mathrm{V}$ at $77 \mathrm{~K}$ along a vertical line at $X=20 \mu \mathrm{m}$ beside the outer n-well sidewall of the test structure. Comparison of calculated electron drift current density between $V_{b e}=0.7 \mathrm{~V}$ at $\mathrm{RT}$ and $V_{b e}=1.1 \mathrm{~V}$ at $77 \mathrm{~K}$ along this line (f) in the $X$-direction and (h) in the $Y$-direction.

formance. A thinner epitaxial-layer thickness will lead to a stronger width dependence of the $I_{c 2}$ with a decrease in its magnitude [9]. In order to further understand the effect of the epitaxial-layer thickness on the $I_{c 2}$ characteristics between low temperature and RT, we consider different guard ring structures with several epitaxial-layer thicknesses by the numerical simulation. Fig. 6(a) shows the three types of ideal doping profiles along the depth direction beneath the guardring well. The epitaxial-layer thicknesses are about $2.5,5$, and $10 \mu \mathrm{m}$. The ideal 5 and $10 \mu \mathrm{m}$ cases have the high/low junction transition regions from 5 to $6 \mu \mathrm{m}$, and from 9 to $11 \mu \mathrm{m}$, respectively. The two structures are identical except the epitaxial-layer thickness. The structure of the ideal 2.5 $\mu \mathrm{m}$ case with an ideal high/low junction, in which transition ranges only $0.5 \mu \mathrm{m}$ wide, is based on a sub-half micron technology as cited in [25]. Fig. 6(b) shows the $I_{c 2}$ versus $W_{g}$ characteristics for the ideal $10 \mu \mathrm{m}$ case. The biases at which the $I_{c 2}$ is measured for the RT and $77 \mathrm{~K}$ cases are chosen to correspond to the same amount of the $I_{e}$. The $I_{c 2}$ at $77 \mathrm{~K}$ is greater than that at RT. Moreover, the slope of the $I_{c 2}$ at $77 \mathrm{~K}$ with low bias is about the same as that at RT and becomes more gradual than that at RT when the $V_{b e}$ bias becomes larger. Fig. 6(c) shows the $I_{c 2}-W_{g}$ characteristics for the ideal $5 \mu \mathrm{m}$ case. For low $V_{b e}$ bias the $I_{c 2}$ exhibits a similar tendency with the experimental results whereas for high $V_{b e}$ bias the behavior resembles that of the ideal $10 \mu \mathrm{m}$ case. Fig. 6(d) illustrates the $I_{c 2}-W_{g}$ characteristics for the ideal $2.5 \mu \mathrm{m}$ case. A similar tendency with the experiments appears for all $V_{b e}$ biases. The $I_{c 2}$ also exhibits a saturation phenomenon due to the dominance of the $I_{s u b}$ [11], [12]. Fig. $6(\mathrm{~d})$ indicates that the saturation property is improved by several orders of magnitude when operated at low temperature. This can be attributed to the better minority-carrier collection capability of the heavily-doped substrate at $77 \mathrm{~K}$ and the positive temperature dependence of the well-junction leakage current as well. 


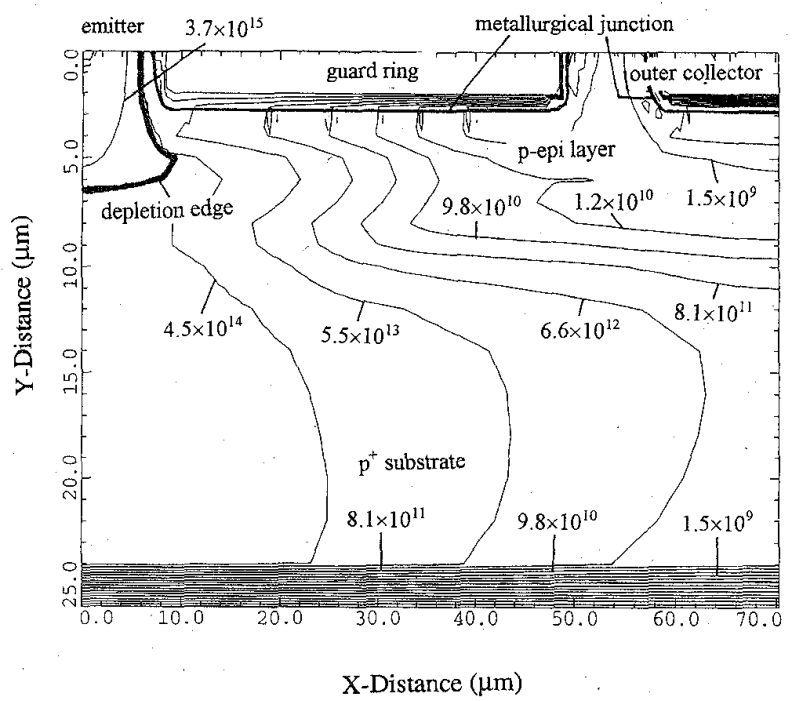

(a)

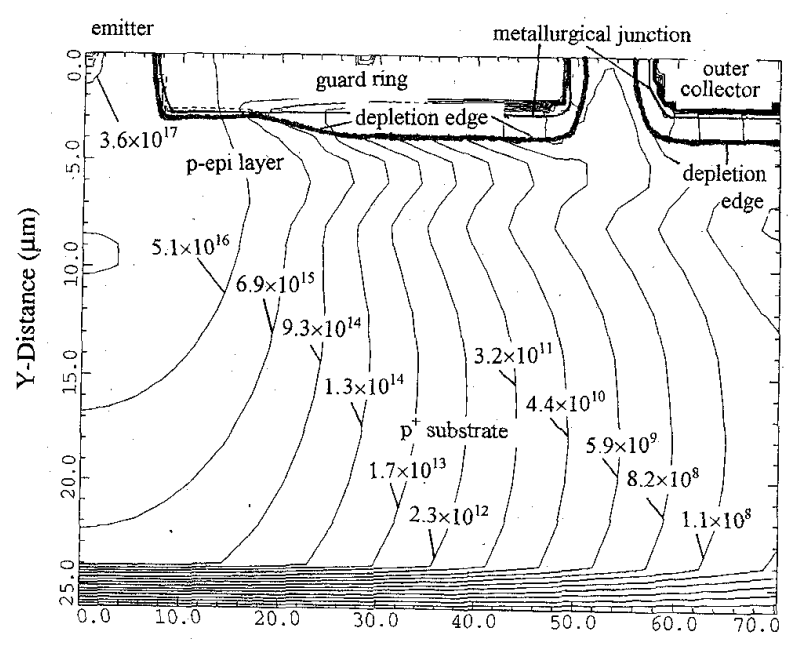

X-Distance $(\mu \mathrm{m})$

(c)

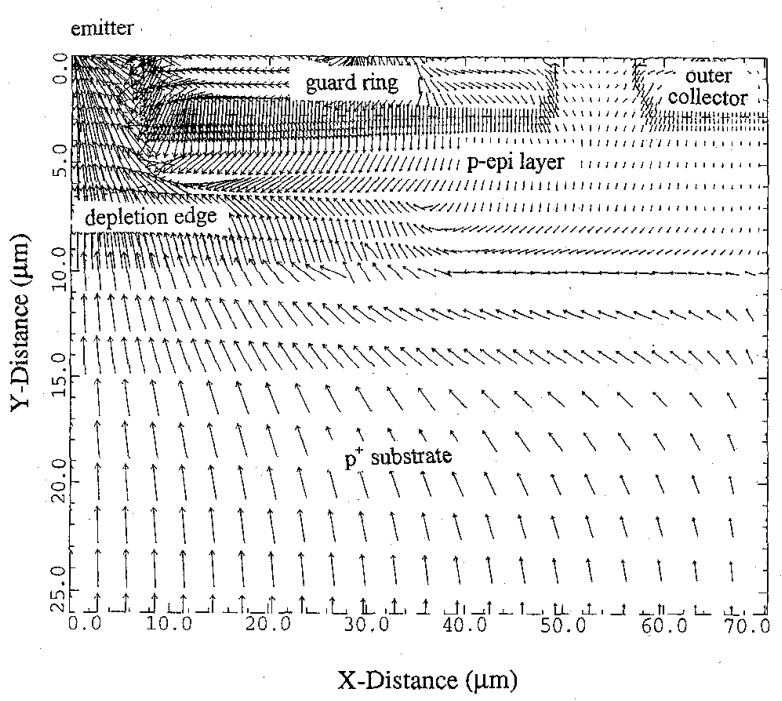

(b)

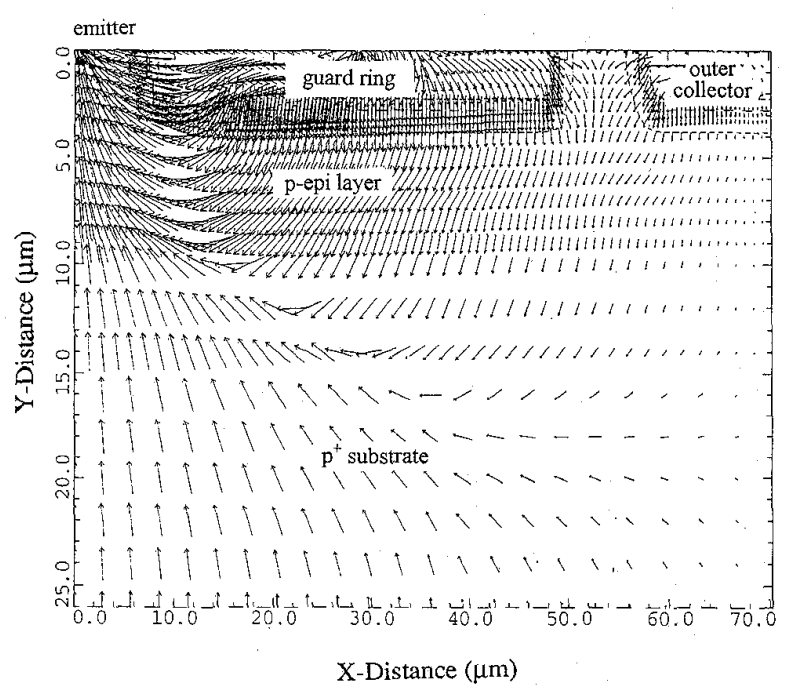

(d)

Fig. 5. The 2-D minority-carrier concentration contours with $W_{g}=40 \mu \mathrm{m}$ (a) at $V_{b e}=1.5 \mathrm{~V}$ for the $77 \mathrm{~K}$ case, (c) at $V_{b e}=1.0 \mathrm{~V}$ for the RT case. The plot of 2-D minority-carrier current vectors with $W_{g}=40 \mu \mathrm{m}(\mathrm{b})$ at $V_{b e}=1.5 \mathrm{~V}$ for the $77 \mathrm{~K}$ case, and (d) at $V_{b e}=1.0 \mathrm{~V}$ for the RT case.

Fig. 7(a)-(d) show the 2-D minority carrier contours and their current flow vectors, respectively, with ideal epitaxiallayer thickness of $10 \mu \mathrm{m}$ and with $W_{g}$ of 20 and $40 \mu \mathrm{m}$ at $77 \mathrm{~K}$. The depletion regions of the two n-wells in the $20 \mu \mathrm{m}$ case are separated and the sandwich layer under the guard ring bottom becomes quasi-neutral because of the thicker epitaxial layer. In the $40 \mu \mathrm{m}$ case the depletion region under the guard-ring well also shrinks partially. This clearly explains the degraded collection capability of the guard-ring well bottom. In addition, high-level injection in the lightly-doped epitaxial layer at $77 \mathrm{~K}$ is severer than that at RT and the high/low junction transition is also sharper than the test key profile. Hence the $I_{c 2}$ for this ideal $10 \mu \mathrm{m}$ case exhibits a weaker width dependence and a greater magnitude than that at RT. Fig. 7(b) and (d) both shows that the lower portion of the current vectors flow horizontally along the sandwich layer, further confirming the uncontrollableness of the guard-ring well. The $I_{c 2}$ of the ideal $5 \mu \mathrm{m}$ case at low bias show a similar tendency to the experimental results owing to the certain similarities of the two structures, i.e., the experimental structure has an effective epitaxial layer of $6 \mu \mathrm{m}$. When $V_{b e}$ bias is close to $2.0 \mathrm{~V}$, although the low-doped epitaxial layer is quite thin, the 2-D minority-carrier contours still reveal the separation of the depletion regions of the two n-wells. Such evidence explains the likeness between the ideal $5 \mu \mathrm{m}$ case with large applied bias and the ideal $10 \mu \mathrm{m}$ case at $77 \mathrm{~K}$. As for the ideal $2.5 \mu \mathrm{m}$ case, its behavior can also be interpreted by the same arguments. In conclusion, these all imply that the structure with a thinner epitaxial layer exhibits more significant width dependence and less magnitude of the $I_{c 2}$ for low temperature operation. 


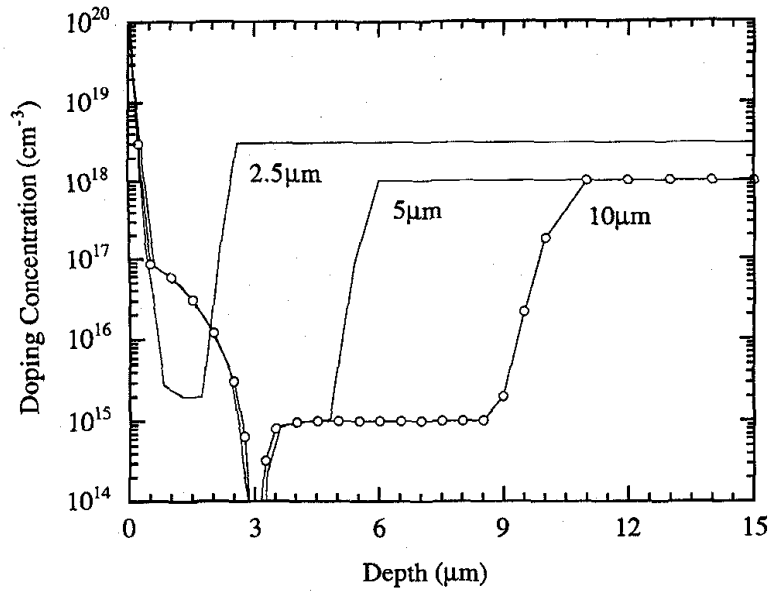

(a)

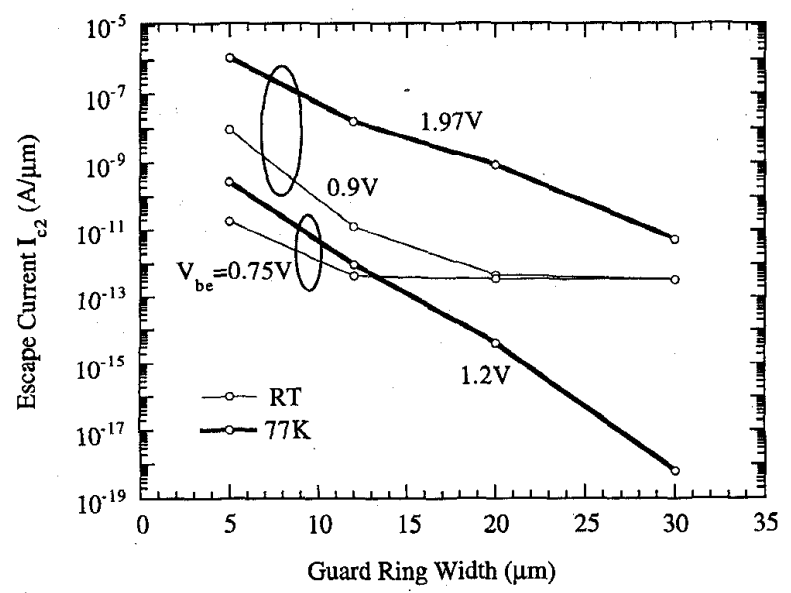

(c)

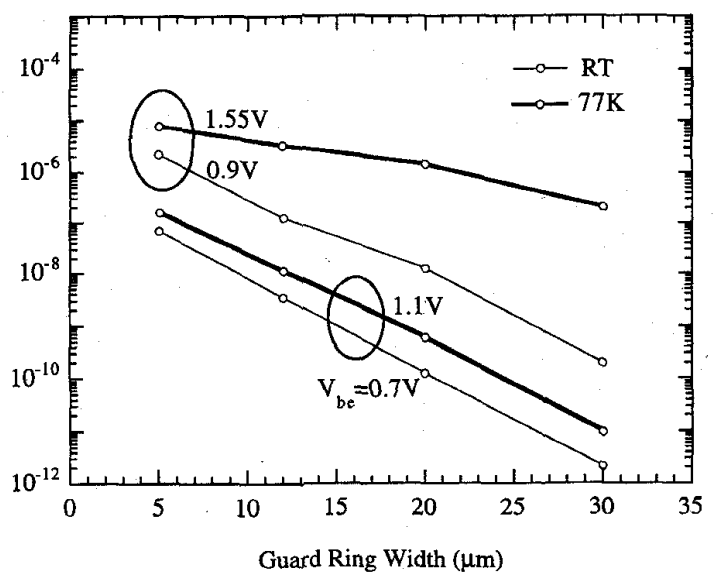

(b)

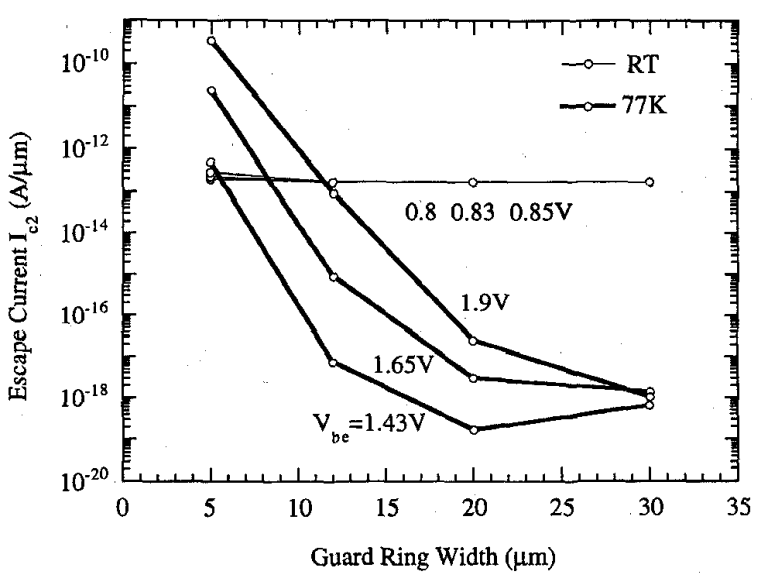

(d)

Fig. 6. (a) The ideal doping profile with the epitaxial layer thicknesses of $2.5,5$ and $10 \mu \mathrm{m}$ along the depth direction under the guard ring. (b) The simulated characteristics of the $I_{c 2}$ as a function of $W_{g}$ at RT and $77 \mathrm{~K}$ for the ideal $10 \mu \mathrm{m}$ case. The $V_{b e}$ biases of the first comparison pair are 0.7 and $1.1 \mathrm{~V}$, the second pair are $0.9 \mathrm{~V}$ and $1.55 \mathrm{~V}$ at RT and $77 \mathrm{~K}$, respectively. (c) The simulated characteristics of the $I_{c 2}$ as a function of $W_{g}$ at RT and $77 \mathrm{~K}$ for the ideal $5 \mu \mathrm{m}$ case. The $V_{b e}$ biases of the first comparison pair are 0.75 and $1.2 \mathrm{~V}$, the second pair are 0.9 and $1.9 \mathrm{~V}$ at RT and $77 \mathrm{~K}$, respectively. (d) The simulated characteristics of the $I_{c 2}$ as a function of $W_{g}$ at RT and $77 \mathrm{~K}$ for the ideal $2.5 \mu \mathrm{m}$ case. The $V_{b e}$ biases of the first comparison pair are 0.8 and $1.43 \mathrm{~V}$, the second pair are 0.83 and $1.65 \mathrm{~V}$, and the third pair are 0.85 and $1.9 \mathrm{~V}$ at RT and $77 \mathrm{~K}$, respectively.

\section{DISCUSSION}

Carrier recombination in the base region requires some discussion. SRH recombination is a strong function of temperature [18], but because of the lack of a fully justified model the equation utilized at RT is used here for low-temperature simulation. The effect of SRH recombination indeed cannot be evaluated quantitatively. Nevertheless, carrier recombination plays an important role only at low-level injection. SRH recombination can be expected to be irrelevant to the increase in the escape current for the narrow $W_{g}$ case. Furthermore, Auger recombination, which is dominant in heavily-doped semiconductors, exhibits rather weak dependence on temperature and its coefficient, which decreases slightly from RT to 77 $\mathrm{K}$, leads to a low recombination rate. This may be one of the factors that contribute to the larger amount of the minority carriers and the current density in the substrate. However, most of the substrate current flows toward the substrate con- tact. Hence, Auger recombination doesn't contribute to the increased escape current.

Base resistance $R_{b}$ typically plays an important role in highlevel injection characteristics. The $R_{b}$ in this test structure is mainly determined by the intrinsic lightly doped epitaxial region between the emitter and the n-well guard ring, and the resistance of the substrate may be neglected because of the highly-doped concentration. The $R_{b}$ of an ordinary vertical BJT at $77 \mathrm{~K}$ actually increases in the ideal diffusion region, but decreases in the high injection owing to more severe conductivity modulation at $77 \mathrm{~K}$ in the base region [5]. In general the $R_{b}$ is influenced by the following factors: conductivity modulation, emitter crowding effect, and base pushout effect. Our specific structure is entirely free from emitter crowding effect as well as base pushout effect [18]. The $R_{b}$ can be controlled only by conductivity modulation in the intrinsic base region. The $R_{b}$ was extracted from solutions of simulation by means of dividing the potential difference 


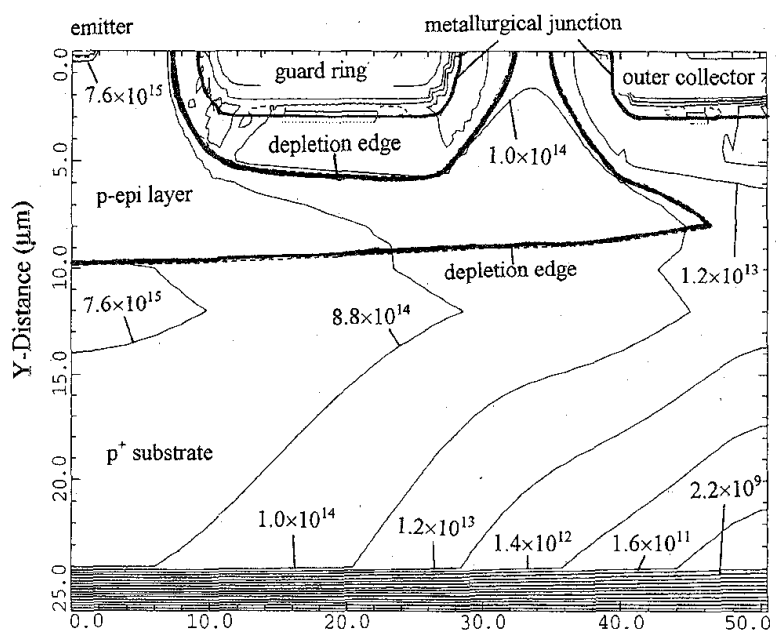

$\mathrm{X}$-Distance $(\mu \mathrm{m})$

(a)

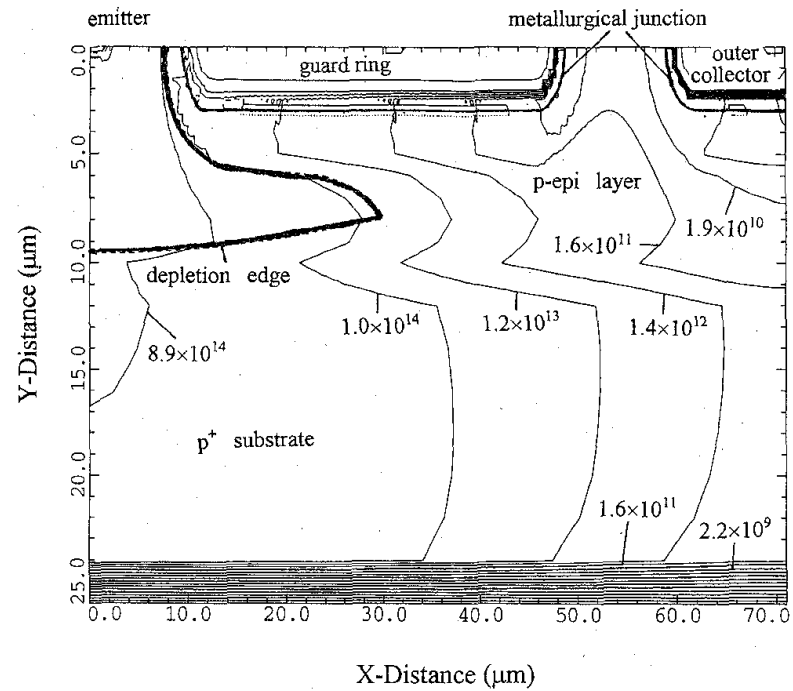

(c)

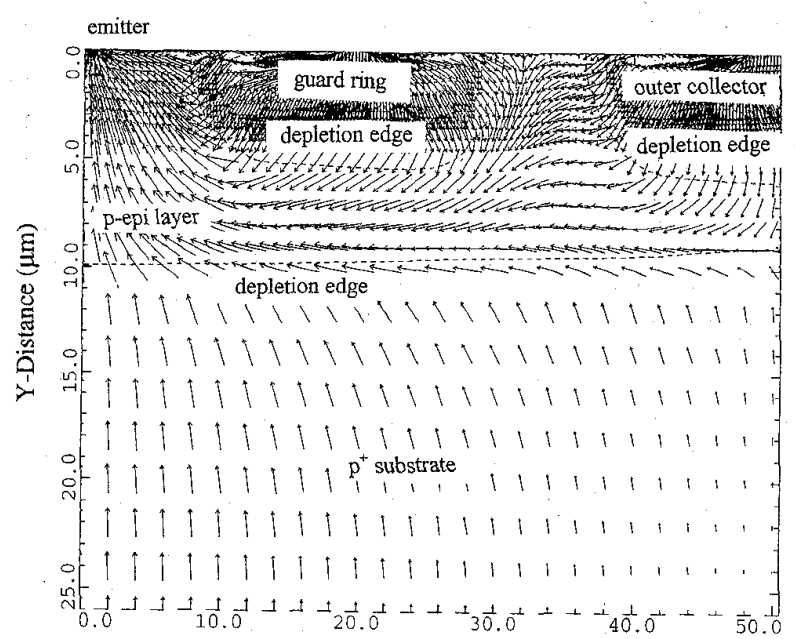

$\mathrm{X}$-Distance $(\mu \mathrm{m})$

(b)

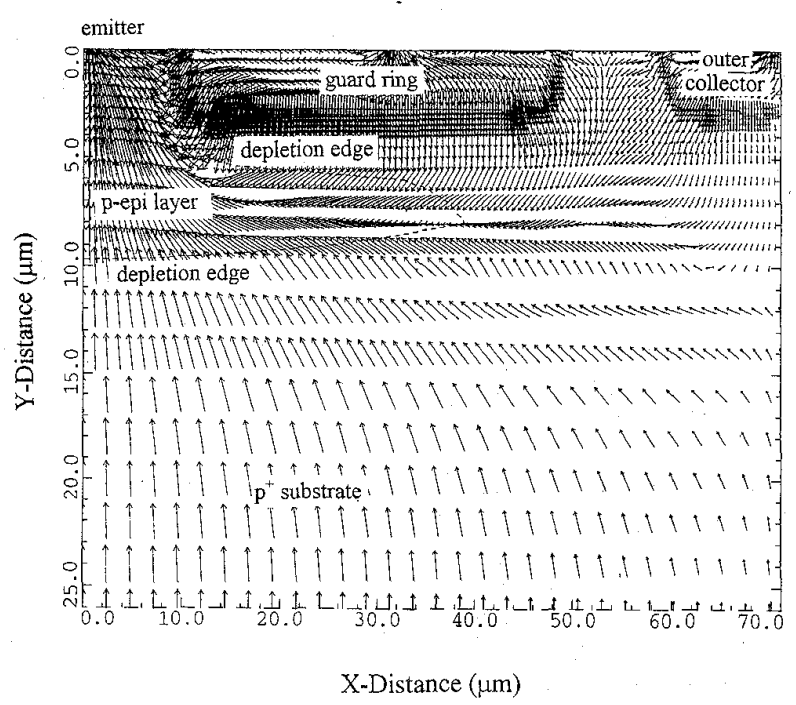

(d)

Fig. 7. The 2-D minority-carrier concentration contour of the ideal $10 \mu \mathrm{m}$ case with $W_{g}=$ (a) 20 and (c) $40 \mu \mathrm{m}$ at $V_{b e}=1.6 \mathrm{~V}$ for $77 \mathrm{~K}$. The plot of 2-D minority-carrier current vector of the ideal $10 \mu \mathrm{m}$ case with $W_{g}=$ (b) 20 and (d) $40 \mu \mathrm{m}$ at $V_{b e}=1.9 \mathrm{~V}$ for $77 \mathrm{~K}$.

in the base region by the base current. It was calculated to have a value which decreases with decreasing temperature in agreement with [5]. In fact, both the reduced $R_{b}$ and the enhanced drift current can be traced back to conductivity modulation in high-level injection.

Epitaxial $\mathrm{p} / \mathrm{p}^{+}$high/low junction at low temperature also exhibits behavior far different from PN junction, as shown in Fig. 8. At low temperature semiconductors are well known to become more degenerate, that is, both $\phi_{f p}$ and $\phi_{f n}$ become larger. Thus built-in potential $\phi_{f p}+\phi_{f n}$ of a PN junction increases as temperature decreases [15]. In contrast, built-in potential of a $\mathrm{p} / \mathrm{p}^{+}$high/low junction at thermal equilibrium exhibits an opposite tendency, as demonstrated in Fig. 8. The Fermi level on the lightly-doped side approaches the valence band as the temperature decreases while on the heavilydoped side the doping is above the Mott transition [18], it is not subject to freezeout and hence no shift in the Fermi level occurs. As a result $\phi_{f p}$ increases, causing more degenerate on the lightly-doped side. The built-in potential of the high/low junctions, which is equal to $E_{g} / 2 q-\phi_{f p}$, is reduced along with the decreased temperature. Therefore, at low temperature minority-carrier reflection ability of the high/low junction diminishes as a consequence of the reduced built-in field. In other words, the substrate absorbs minority carriers more effectively at low temperature. This phenomenon can clearly be seen from the minority-carrier contour lines in Fig. 3(a) and (b), which spread more widely into the heavilydoped substrate. This is also shown in Fig. 4(f) and (h), in which a greater amount of the minority-carrier current at low temperature is observed: Hence the lowering of the built-in potential is also a contributing factor for the strong width dependence of the $I_{c 2}$. 


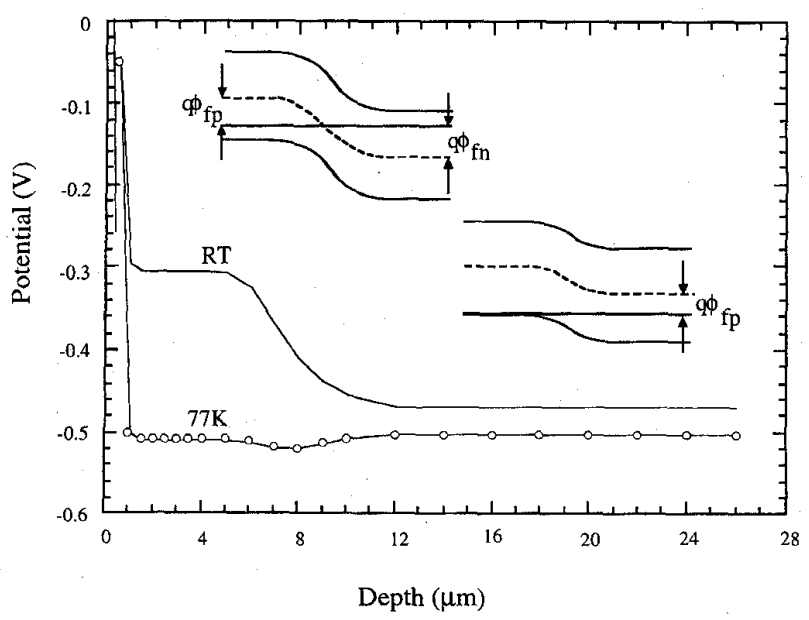

Fig. 8. Comparison of potential distribution at thermal equilibrium between RT and $77 \mathrm{~K}$ along the depth direction at $X=0.0 \mu \mathrm{m}$. The inserted graphs illustrate the energy band diagrams of the PN junction and $\mathrm{p}^{+} / \mathrm{p}$ high/low junction.

Finally, several design considerations of the guard ring for better low-temperature operation may be drawn from the above experimental and simulated results. In spite of a special latch up-free case reported in [4], latch up still exists generally in CMOS operated around $77 \mathrm{~K}$ [26], i.e., reducing temperature is just one of the possible parameters for latch up elimination design. Therefore, the design issues addressed below may provide further information for total latch up suppression or at least other leakage prevention at low temperature. First of all, the lightly-doped epitaxial layer on a heavily-doped substrate becomes more essential at low temperature than at RT. The epitaxial-layer thickness should be as thin as possible to eliminate the $I_{s a n}$, which is usually the dominant component of the $I_{c 2}$. From the above results the epitaxial-layer thickness should be kept at least below $10 \mu \mathrm{m}$. The limiting epitaxiallayer thickness is equal to the n-well depth such that the $I_{\text {san }}$ vanishes totally. In view of the better sinking capability of the heavily-doped substrate, minority-carrier guard rings may be accompanied by majority-carrier guard rings connected with the substrate [27]. In such circumstance the majority-carrier guard rings can collect not only majority carriers but also minority carriers the same as the heavily-doped substrate does. Next, if the epitaxial layer thickness is around $10 \mu \mathrm{m}$, the $W_{g}$ should be kept larger, for example, about $20 \mu \mathrm{m}$ for better low-temperature performance. As the epitaxial-layer thickness decreases the minimum $W_{g}$ for better performance can be reduced. To sum up, these considerations for enhanced lowtemperature performance have a similar trend as those at RT. This similar tendency is beneficial and convenient for both temperature operations by using only one design structure.

\section{CONCLUSION}

The experiments and simulations concerning the performance of the minority-carrier well-type guard ring have been compared between RT and $77 \mathrm{~K}$. The comparisons under the same amount of the minority carrier density give a more deep insight. The escape probability of a narrow guard ring is about one order of magnitude higher at $77 \mathrm{~K}$ and its width dependence becomes more significant than that at RT. This property has been investigated in detail by the numerical simulations. Design considerations have also been drawn from these experiments and simulations. A lightly-doped epitaxial layer on a heavily-doped substrate exhibits more significance in the guard ring efficiency at low temperature, and its thickness should be kept as thin as possible.

\section{ACKNOWLEDGMENT}

The authors would like to thank P.-N. Tseng of Taiwan Semiconductor Manufacturing Company for providing the test devices designed by us. They are also grateful to the reviewers for their helpful comments and suggestions.

\section{REFERENCES}

[1] F. H. Gaensslen, V. L. Rideout, E. J. Walker, and J. J. Walker, "Very small MOSFET'S for low-temperature operation," IEEE Trans. Electron Devices, vol. ED-24, p. 218, 1977.

[2] S. Hanamura, M. Aoki, T. Masuhara, O. Minato, Y. Saki, and T. Hayashida, "Low-temperature CMOS $8 \times 8$ bit multipliers with sub10-ns speeds," IEEE Trans. Electron Devices, vol. ED-34, p. 94, 1987.

[3] J. Y. Sun, Y. Taur, R. H. Dennard, and S. P. Klepner, "Submicrometer channel CMOS for low-temperature operation," IEEE Trans. Electron Devices, vol. ED-34, p. 19, 1987.

[4] E. Sangiorgi, P. F. Bechtold, W. Fichtner, R. L. Johnston, and M. R. Pinto, "Temperature-dependence of latch up phenomena in scaled CMOS structures," IEEE Electron Device Lett., vol. EDL-7, p. 28, 1986.

[5] H. Satake and T. Hamasaki, "Low-temperature (77 K) BJT model with temperature dependences on the injected condition and base resistance," IEEE Trans. Electron Devices, vol. 37, p. 1688, 1990.

[6] N. West and K. Eshraghian, Principles of CMOS VLSI Design-A Systems Perspective. Reading, MA: Addison-Wesley, 1985, p. 60.

[7] J. Quinke, "Analysis of latch up neighborhood effects in VLSI CMOS input/output stages," Microelectron. Reliab., vol. 30, p. 105, 1990.

[8] R. R. Troutman, "Epitaxial layer enhancement of n-well guard rings for CMOS circuits," IEEE Electron Device Lett., vol. EDL-4, p. 438, 1983.

[9] M. J. Chen and C. Y. Wu, "A simplified computer analysis for N-well guard ring efficiency in CMOS circuits," Solid-State Electron., vol. 30 , p. 879,1987

[10] M. J. Chen, C. Y. Huang, P. N. Tseng, N. S. Tsai, and C. Y. Wu, "Design model for minority-carrier well-type guard rings in CMOS circuits," in Proc. IEEE Custom IC's Conf., 1991, p. 4.5.1.

[11] M. J. Chen, C. Y. Huang, and P. N. Tseng, "Design formulation for minority-carrier well-type guard rings in CMOS circuits," IEE Proc.-G Circuits, Dev. Sys., vol. 140, p. 182, 1993.

[12] C. Y. Huang and M. J. Chen, "Design model and guideline for minoritycarrier well-type guard rings in epitaxial CMOS," IEEE Trans. Electron Devices, vol. 41, p. 1806, 1994.

[13] E. Hackbarth and D. D. Tang, "Inherent and stress-induced leakage in heavily-doped silicon junctions," IEEE Trans. Electron Devices, vol. 35 , p. $2108,1988$.

[14] S. M. Sze, Physics of Semiconductor Devices, 2nd ed. New York: Wiley, 1982.

[15] C. Nguyenduc, S. Cristoloveanu, and G. Ghibaudo, "Low-temperature mobility behavior in submicron MOSFET's and related determination of channel length and series resistance," Solid-State Electron., vol. 29, p. 1271, 1986.

[16] MEDICI 1.0 User's Manual. CA: Technology Modeling Associates, Inc., 1992.

[17] S. Selberherr, "MOS device modeling at $77 \mathrm{~K}$," IEEE Trans. Electron Devices, vol. 36 , p. 1464, 1989.

[18] M. Chrzanowska-Jeske and R. C. Jaeger, "BILOW-simulation of low temperature bipolar device behavior," IEEE Trans. Electron Devices, vol. 36 , p. $1475,1989$.

[19] Y, W. Chen and J. B. Kuo, "Two-dimensional analysis of a BiNMOS transistor operating at $77 \mathrm{~K}$ using a modified PISCES program," IEEE Trans. Electron Devices, vol. 39, p. 348, 1992.

[20] A. K. Kapoor and T. S. Jayadev, "Operation of poly bipolar transistors near liquid helium temperature $(9 \mathrm{~K})$," IEEE Electron Device Lett., vol. 9 , p. 177,1988 . 
[21] J. W. Slotboom, "The pn product in silicon," Solid-State Electron., vol. 20, p. $279,1977$.

[22] J. Dziewior and W. Schmid, "Auger coefficients for highly-doped and highly-excited silicon," Appl. Phys. Lett., vol. 31, p. 346, 1977.

[23] D. M. Caughey and R. E. Thomas, "Carrier mobilities in silicon empirically related to doping and field," Proc. IEEE, vol. 52, p. 2192, 1967.

[24] A. K. Hening, N. N. Chan, J. T. Watt, and J. D. Plummer, "Substrate current at cryogenic temperatures: Measurements and a two-dimensional model for CMOS technology," IEEE Trans. Electron Devices, vol. ED34, p. $64,1987$.

[25] R. A. Chapman, J. W. Kuehne, P. S-H. Ying, W. F. Richardson, A. R. Peterson, A. P. Lane, I.-C. Chen, L. Velo, C. Blanton, M. Moslehi, and J. Paterson, "High performance sub-half micron CMOS using rapid thermal processing," in IEDM Tech. Dig., 1991, p. 101.

[26] J. G. Dooley and R. C. Jaeger, "Temperature dependence of latch up in CMOS circuits," IEEE Electron Device Lett., vol. EDL-5, p. 41, 1984.

[27] R. R. Troutman, Latch up in CMOS Technology: The Problem and Its Cures. Boston, MA: Kluwer, 1986.

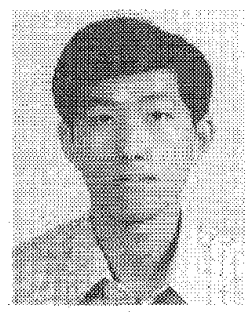

Chih-Yao Huang (S'87-M'95) was born in Hsinchu, Taiwan, R.O.C., on May 31, 1963. He received the B.S. degree in electrical engineering from the National Tsing-Hua University, Hsinchu, in 1985, and the M.S. and Ph.D. degrees in electronics engineering from the National ChiaoTung University, Hsinchu, in 1987 and 1994, respectively.

From 1987 to 1989 , he served in the Chinese Air Force, and from 1991 to 1993 , he was a part-time lecturer in the Institute of Electronics, National Chiao-Tung University. In 1995, he joined Mosel/Vitelic Inc., Hsinchu, working on the analysis and design of submicron semiconductor devices.

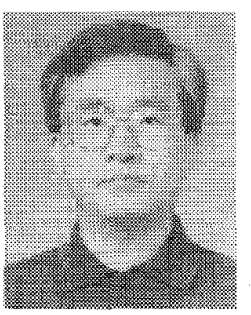

Ming-Jer Chen (S'77-M'86) was born in Taiwan, R.O.C., on April 1, 1954. He received the B.S. degree with highest honors from the National Cheng-Kung University in 1977, and the M.S. and Ph.D. degrees in electrical engineering from the National Chiao-Tung University (NCTU), Hsinchu, in 1979 and 1985, respectively. His doctoral work involved the modeling and prediction of CMOS latch-up.

From 1979 to 1980 , he worked at the Telecommunication Laboratories establishing a multiproceesor distributed system. From 1985 to 1986 , he conducted post-doctoral research on CMOS latch-up at NCTU. From 1986 to 1992, he was an associate professor and in 1993 became a professor, Department of Electronics Engineering, NCTU. From 1987 to 1992, he set up a series of design rules for the Taiwan Semiconductor Manufacturing Company. His current research interests include deep submicron reliability, low power integrated circuits, and subthreshold CMOS and BiCMOS technologies for new applications. He holds five Taiwan patents and two U.S. patents in the above fields.

Dr. Chen has served as a reviewer for international journals such as IEEE Electron Device LetTers, IEEE Transactions on Electron DEvices, Solid State Electronics, and the Journal of the Chinese Institute of Engineers. He is a member of Phi Tau Phi.

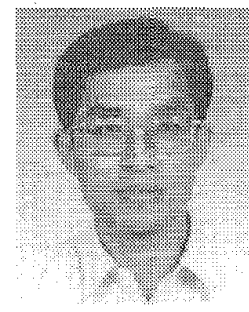

Jeng-Kuo Jeng received the B.S. degree in electrophysics in 1982, and the M.S. degree in electronics engineering in 1990, from the National ChiaoTung University, Hsinchu, Taiwan. He joined the ERSO/TTRI, Hsinchu, in 1984, where he was an integrated circuits reliability and failure analysis engineer in the QRA group. He joined Vanguard Co. Hsinchu, in 1994, and is currently a senior failure analysis engineer for memory products. His technical interests include reliability physics in DRAM and ESD in particular.

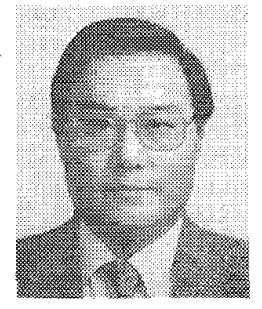

Ching-Yuan Wu (M'72) was born in Taiwan, R.O.C., on March 18, 1946. He received the B.S. degree from the Department of Electrical Engineering, National Taiwan University in 1968, and the M.S. and Ph.D. degrees from the State University of New York (SUNY) at Stony Brook, in 1970 and 1972 , respectively.

During the 1972-1973 academic year, he was appointed as a Lecturer, Department of Electrical Sciences, SUNY-Stony Brook. During the 1973-1975 academic years, he was a Visiting Associate Professor, National Chiso-Tung University (NCTU), Hsinchu, Taiwan, R.O.C. In 1976, he became a Full Professor in the Department of Electronics and the Institute of Electronics, NCTU. At NCTU, he was the Director of Engineering Laboratories and Semiconductor Research Center from 1974 to 1980; the Director of the Institute of Electronics from 1978 to 1984; and the Dean, College of Engineering, from 1984 to 1990. He was a principal investigator of the National Electronics Mass PlanSemiconductor Devices and Integrated-Circuit Technologies from 1976 to 1979. His research activities have been in semiconductor device physics and modelings, Integrated circuit designs and technologies. His present research areas focus on the development of efficient 2-D and 3-D simulators for deep-submicrometer semiconductor devices, design rules and optimization techniques for deep-submicrometer CMOS devices, and key technologies for deep-submicrometer CMOS devices. He has published more than 180 papers in the semiconductor field and has served as a reviewer for international journals such as IEEE ELectron Device LetTers, IEEE Transactions on EleCtron Devices, Solid-State Electronics, etc.

Dr. Wu is a member of the Honorary Editorial Advisory Board of SolidState Electronics and is a board member of the Chinese Engineering Society. He received the Academic Research Award in Engineering from the Ministry of Education (MOE), in 1979, the outstanding Scholar award from the Chinese Educational and Cultural Foundation, in 1985. From 1982 to 1995, he has received the outstanding research Professor fellowship from the Ministry of Education and the National Science Council (NSC), ROC. In 1992, he received the Distinguished Engineering Professor Medal Award from the Chinese Engineering Society. From 1982 to 1988, he was a Coordinator of the National Microelectronics Researches and High-Level Man-Power Education Committee, National Science Council, R.O.C. He has been the Research Consultant of the Electronics Research and Service Organization (ERSO), ITRI; a member of the Academic Review Committee, the Ministry of Education; and the chairman of the Technical Review Committee on information and Microelectronics Technologies, the Ministry of Economic Affairs. 